\title{
Non-Hodgkin's lymphoma with uncommon clinical manifestations: A case report
}

\author{
YU-LONG CAI, XIAN-ZE XIONG, JIONG LU, YI-XIN LIN and NAN-SHENG CHENG \\ Department of Bile Duct Surgery, West China Hospital, Sichuan University, Chengdu, Sichuan 610041, P.R. China
}

Received September 23, 2014; Accepted June 2, 2015

DOI: $10.3892 / \mathrm{ol} .2015 .3493$

\begin{abstract}
Extranodal lymphoma occurs in $\sim 40 \%$ of all patients with lymphoma and has been described in virtually all organs and tissue. However, diffuse large B-cell lymphoma (DLBCL), which is the most common histological subtype of non-Hodgkin's lymphoma (NHL), primarily arising in the retroperitoneal region has been rarely reported. Primary retroperitoneal lymphoma without renal or ureteral involvement affecting the genitourinary system has not been reported until now. In this article, we report the rare case of a young female suffering with primary DLBCL located simultaneously in the retroperitoneal and gastrointestinal region. The first sign of this disease was renal colic. Pathological assessment was performed using immunohistochemistry staining, revealing positivity for CD20 and Ki67. DLBCL was the final confirmed diagnosis. Obtaining a definitive histological diagnosis by surgery and using exactly chemotherapy played an essential role in the treatment of our patient. This case serves as a reminder to include the differential diagnosis of primary retroperitoneal NHL when a patient presents with a retroperitoneal mass and the first symptom is renal colic.
\end{abstract}

\section{Introduction}

Extranodal lymphoma occurs in $\sim 40 \%$ of all patients with lymphoma and has been described in virtually all organs and tissue (1). Extranodal disease is more common with non-Hodgkin's lymphoma (NHL) (2), and diffuse large B-cell lymphoma (DLBCL) is the most common histological NHL subtype in adults, accounting for $25 \%$ of all NHL cases (3). Thus, it is known that gastrointestinal DLBCL is the most frequent form of extranodal lymphoma (4). However, DLBCL primarily arising in the retroperitoneal region has been rarely reported. The largest series on retroperitoneal DLBCL was published by

Correspondence to: Professor Nan-Sheng Cheng, Department of Bile Duct Surgery, West China Hospital, Sichuan University, 37 Guo Xue Xiang, Chengdu, Sichuan 610041, P.R. China

E-mail: nanshengcheng2012@163.com

Key words: diffuse large B-cell lymphoma, renal colic, retroperitoneal neoplasms, pathology, surgical
Pileri et al in 2001 (5). Moreover, to the best of our knowledge, primary retroperitoneal lymphoma without renal and ureteral involvement affecting the genitourinary system has not been reported until now.

Here, we report the extremely rare case of a young female suffering with primary DLBCL located in the retroperitoneal and gastrointestinal region simultaneously. Unusually, the first symptom of this disease was renal colic. Written informed consent was obtained from the patient for inclusion in the present study.

\section{Case report}

Case presentation. A 33-year-old female presented with a 2-month history of renal colic and abdominal pain, which became aggravated at night. No fever was noted, but there was hematuria when the pain occurred. The patient's weight remained unchanged. Her family history was not contributory. Surgical history included two Caesarean sections 13 and 9 years prior.

Physical examination revealed an ill-defined mass in the right lower hypogastrium and tenderness in the abdomen, but without abdominal distention. There were no enlarged or palpable lymph nodes. The remaining systemic examination was not significant. The peripheral blood count was unremarkable (hemoglobin $109 \mathrm{~g} / 1$, red blood cell count $3.81 \times 10^{12} / 1$, white blood cell count $3.05 \times 10^{9} / 1$, and platelet count $\left.230 \times 10^{9} / 1\right)$. The peripheral blood smear revealed no immature cells $(66.2 \%$ neutrophils, $25.6 \%$ lymphocytes, $7.2 \%$ monocytes, $1.0 \%$ eosinophils and $0.0 \%$ basophils). Liver and renal functional tests, bilirubin and electrolytes were normal. Serum tumor markers were negative with the exception of CA-125 values of $63.88 \mathrm{U} / \mathrm{ml}$ (normal value, $<35 \mathrm{U} / \mathrm{ml}$ ). The remaining laboratory tests were all within the normal limits.

Imaging. A normal chest X-ray was obtained. An abdominopelvic contrast-enhanced computed tomography (CT) scan (Fig. 1) revealed: i) A bulky soft-tissue dense mass in the middle of the ascending colon and superior to the ileocecum; heterogeneous enhancement following enhanced scan; thickened anterior of the renal fascia of the right kidney and local parietal peritoneum. ii) Multiple renal cysts in both kidneys. The CT scan did not indicate any bowel involvement, distant metastasis or abdominal lymph node enlargement. 


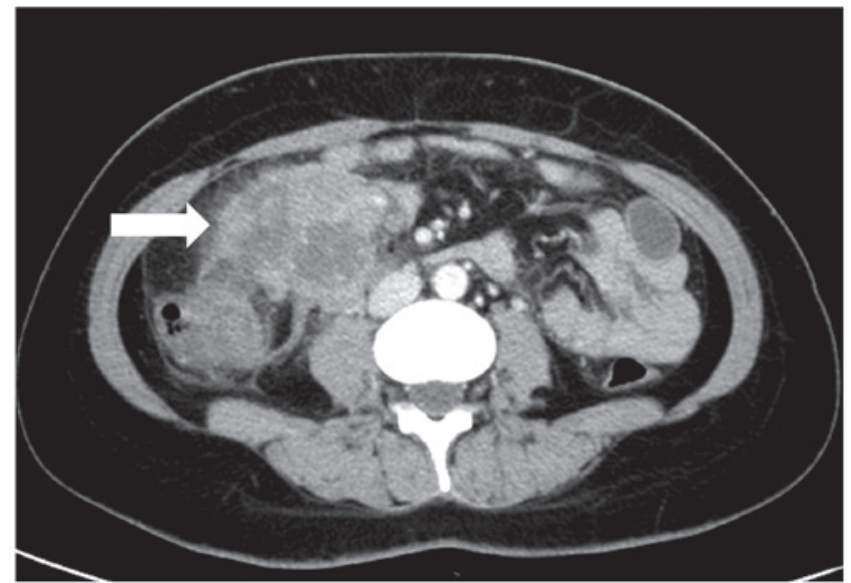

Figure 1. Abdominopelvic contrast-enhanced computed tomography scan revealed a bulky soft-tissue dense mass (white arrow) in the middle of the ascending colon and superior to the ileocecum. Heterogeneous enhancement was noted following the enhanced scan.

The abdominal ultrasound did not reveal any coexisting lesion in the hepato-pancreato-biliary system. Single-photon emission computed tomography (SPECT) renal imaging (99mTc-DTPA) revealed that the glomerular filtration rate was slightly decreased and the upper urinary tract had unobstructed drainage in the two kidneys.

Surgical treatment. Since the tumor had no distant involvement and there was no evidence of worsening symptoms (renal colic and abdominal pain), the patient underwent surgical resection. Intra-operative findings were as follows: no ascites were in the abdominal cavity; no dilation of the small and large bowel; the mass was predominantly located in the right mesocolon and retroperitoneal region, and extended to the distal ileum, ascending colon and the beginning of the transverse colon. Intra-operative biopsy and frozen section study indicated malignancy but did not confirm the tumor type. Complete excision was performed, retaining the right kidney and right ureter due to their lack of involvement. Side-to-side anastomosis of the transverse colon and ileum was used. The patient had an uneventful postoperative recovery. She was discharged from the surgical ward and referred to the hematology clinic for additional evaluation and adjuvant chemotherapy.

Pathological evaluation. The tumor consisted of two masses. The first mass (measuring 9x8x7 cm) was located in the retroperitoneal region, and the second (measuring $2.5 \times 2 \times 2 \mathrm{~cm}$ ) was located in the mucosa of the ileum, involving the submucosa and muscularis layers as well as the serosa. There was no association between the two masses.

Pathological assessment was performed using immunohistochemistry staining, which revealed positivity for CD20 and Ki67) (Fig. 2). DLBCL was the final confirmed diagnosis.

Follow-up. The patient's general condition remained good and she went on to receive CHOP chemotherapy. After 3 months of follow-up, no postoperative complications were identified.
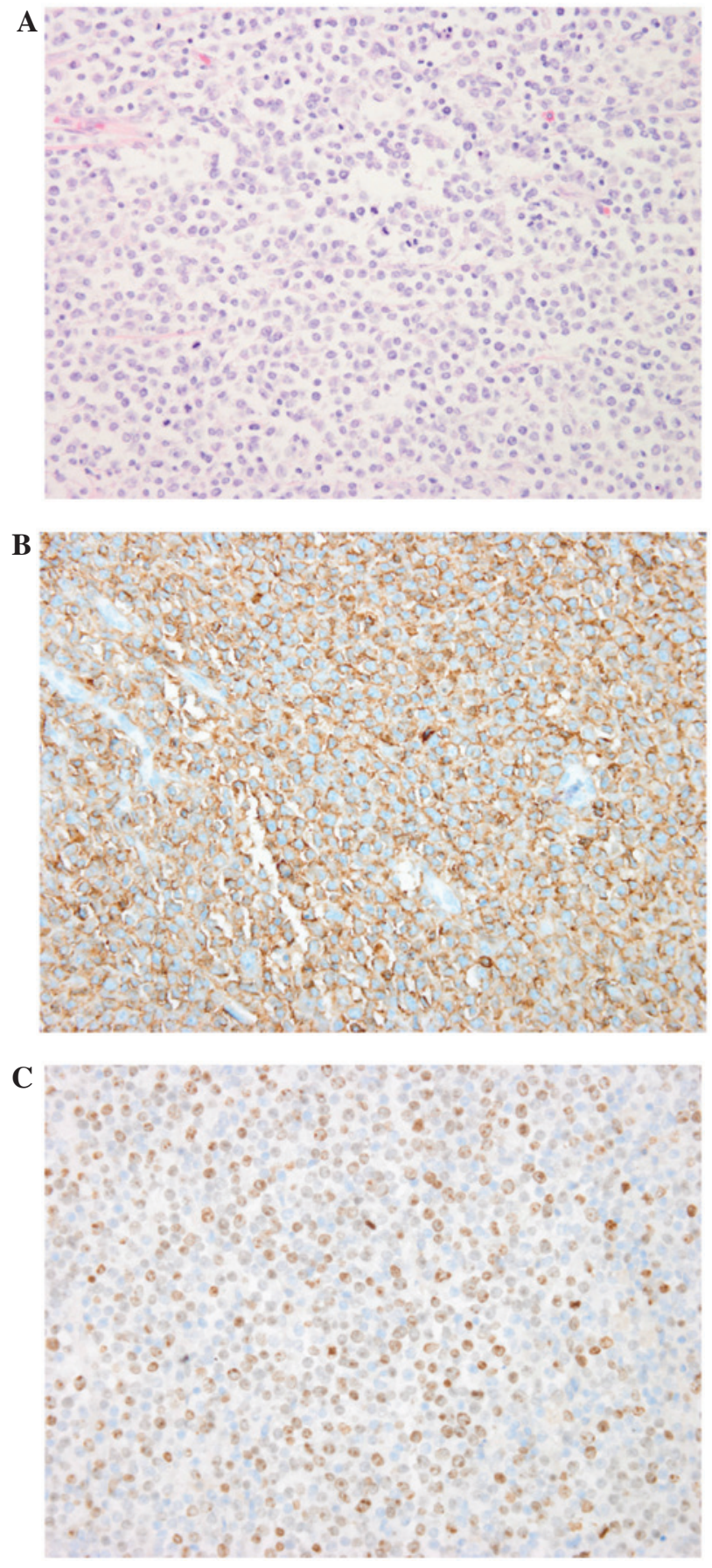

Figure 2. Pathological assessment: (A) hematoxylin and eosin staining: diffuse infiltration of neoplastic lymphoid cells (magnification, x400); (B) tumor cells immunoreactive for B-cell marker CD20 (magnification, x400); (C) tumor cells expressing a high Ki67 index (magnification, $\mathrm{x} 400$ ).

\section{Discussion}

DLBCL is the most common type of lymphoma worldwide (6). However, NHL rarely presents with a retroperitoneal or pelvic mass; during post-mortem studies of NHL patients an incident of less than $1 \%$ incidence was noted (7). Due to the uncommon anatomical location, the diagnosis and subsequent management of these patients tend to be difficult. The initial presentation of NHL varies depending on the subtype and involved area, with symptoms including enlarged palpable lymphadenopathy, B-symptoms (fever, weight loss, night 
sweats), and symptoms secondary to compression of adjacent structures. This is a unique case of retroperitoneal DLBLC, in which the first manifestation was renal colic.

Renal colic mainly occurs in patients with renal or ureteral calculus. Based on the findings of the abdominal CT, abdominal X-ray and SPECT renal imaging, our patient did not suffer with renal or ureteral calculus. Certain studies have reported that the genitourinary system may be affected by retroperitoneal NHL. Domazetovski et al (8) presented a case of acute renal failure in a patient with DLBLC, and Jaeger et al (9) reported DLBLC in a male presenting with ureteral stricture. However, the majority of cases have primary renal lymphoma or renal involvement. Renal colic alone without genitourinary involvement, as observed in our patient, is extremely rare, and could only be confirmed by surgery in our case. When the patient initially presented at our hospital, we considered that malignant retroperitoneal tumors account for $\sim 0.1 \%$ of all malignancies (10) and are more common than benign tumors in the retroperitoneal space (11). As the symptom of renal colic was increasing, it was speculated that there was a high possibility of renal involvement. It is known that the most effective treatment is surgical removal of the tumors, with the exception of chemosensitive tumors, and that a definitive diagnosis can usually be made from the surgical specimens (12). Thus, considering the patient's wishes, surgery was performed.

Unexpectedly, the tumors were located not only in the retroperitoneal region, but also in the ileum. The latter was relatively small, therefore no signs or symptoms had been noted. According to our pathological evaluation, there was no association between the two masses. Literature regarding this condition is lacking, thus a reasonable explanation may be the variety of extranodal lymphoma. Moreover, surgery indicated that the tumor did not infiltrate the renal or ureteral areas. Thus, renal colic was determined to have been the result of compression.

Although CT is the diagnostic modality of choice (13), magnetic resonance imaging (MRI) offers superior soft-tissue contrast in comparison with CT. Indeed, a variety of contrast mechanisms have previously been explored for the characterization of lymphoma in the retroperitoneum, including T2-weighted imaging, diffusion-weighted imaging and dynamic contrast-enhanced imaging (14). The absence of MRI is a limitation in our diagnostic process. In the study of Tambo et al (12), a clinicopathological review of 46 primary retroperitoneal tumors identified that MRI imaging diagnosis prior to surgery was compatible with the histological diagnosis in only 26 patients (57\%), and all six malignant lymphoma patients underwent biopsy or surgical resection. Therefore, if clinical diagnosis cannot be determined by MRI, definitive histological diagnosis by biopsy or tumor resection is required in order to determine the appropriate treatment.

Currently, CHOP therapy is the treatment of choice for DLBLC patients. Since rituximab is a chimeric anti-CD20 IgG1 monoclonal antibody which is a cell surface protein that occurs almost exclusively in mature B-cells, the combination of rituximab and $\mathrm{CHOP}$ is likely become the standard for treating patients with DLBLC (15). The prognosis has improved in recent years owing to the development of various aggressive chemotherapeutic regimens depending on the histological type, stage and age of each patient. Therefore, a definitive histological diagnosis is essential for patients with DLBLC. Our patient did not present with any specific indications for the diagnosis of this rare tumor as the initial manifestation was renal colic. Surgery has a key role in establishing a definitive diagnosis.

Primary retroperitoneal DLBLC has a variable and non-specific presentation and may resemble other neoplastic or inflammatory conditions. Manifestations, laboratory data and imaging alone may initially lead to an incorrect diagnosis. Obtaining a definitive histological diagnosis by surgery and using appropriate chemotherapy played an essential role in the recovery of our patient. This case study indicates the significance of including a differential diagnosis of primary retroperitoneal NHL in patients presenting with a retroperitoneal mass where the first sign of this disease is renal colic.

\section{References}

1. Metser U, Goor O, Lerman H, Naparstek E and Even-Sapir E: PET-CT of extranodal lymphoma. AJR Am J Roentgenol 182: 1579-1586, 2004.

2. Vinnicombe SJ and Reznek RH: Extranodal manifestations of lymphoma. Imaging 11: 240-268, 1999.

3. Morton LM, Wang SS, Devesa SS, Hartge P, Weisenburger DD and Linet MS: Lymphoma incidence patterns by WHO subtype in the United States, 1992-2001. Blood 107: 265-276, 2006.

4. d'Amore F, Christensen BE, Brincker H, Pedersen NT, Thorling K, Hastrup J, Pedersen M, Jensen MK, Johansen P, Andersen E, et al; Danish LYFO Study Group: Clinicopathological features and prognostic factors in extranodal non-Hodgkin lymphomas. Eur J Cancer 27: 1201-1208, 1991.

5. Pileri SA, Zinzani PL, Ascani S, Orcioni GF, Gamberi B, Piccioli M, Sabattini E, Poggi S, Piccaluga PP and Falini B: Diffuse large B-cell lymphoma with primary retroperitoneal presentation: Clinico-pathologic study of nine cases. Ann Oncol 12: 1445-1453, 2001.

6. Hunt KE and Reichard KK: Diffuse large B-cell lymphoma. Arch Pathol Lab Med 132: 118-124, 2008.

7. Fulignati C, Pantaleo P, Cipriani G, Turrini M, Nicastro R, Mazzanti R and Neri B: An uncommon clinical presentation of retroperitoneal non-Hodgkin lymphoma successfully treated with chemotherapy: a case report. World J Gastroenterol 11: 3151-3155, 2005.

8. Domazetovski I, Jovanovic R, Kostadinova-Kunovska S, Duganovska S, Labachevski B, Nikolov I, Ivanovski N, Sikole A and Petrushevska G: Acute renal failure in a patient with diffuse large B-cell lymphoma: case report. Prilozi 33: 231-238, 2012.

9. Jaeger CD, McAlvany KL, Zingula SN, Kramer SA and Granberg $\mathrm{CF}$ :Diffuse large B-cell lymphoma in an adolescent male presenting as ureteral stricture. Case Rep Radiol 2014: 239-345, 2014.

10. Heslin MJ, Lewis JJ, Nadler E, Newman E, Woodruff JM, Casper ES, Leung D and Brennan MF: Prognostic factors associated with long-term survival for retroperitoneal sarcoma: Implications for management. J Clin Oncol 15: 2832-2839, 1997.

11. Arlen M and Marcove RC (eds): Retroperitoneal sarcomas. In: Surgical Management of Soft Tissue Sarcomas. W.B. Saunders, Philadelphia, PA, pp203-232, 1987.

12. Tambo M, Fujimoto K, Miyake M, Hoshiyama F, Matsushita C and Hirao Y: Clinicopathological review of 46 primary retroperitoneal tumors. Int J Urol 14: 785-788, 2007.

13. Karaosmanoglu D, Karcaaltincaba M, Oguz B, Akata D, Ozmen $\mathrm{M}$ and Akhan O: CT findings of lymphoma with peritoneal, omental and mesenteric involvement: peritoneal lymphomatosis. Eur J Radiol 71: 313-317, 2009.

14. Nakayama T, Yoshimitsu K, Irie H, Aibe H, Tajima T, Shinozaki K, Nishie A, Asayama Y, Kakihara D, Matsuura S, et al: Usefulness of the calculated apparent diffusion coefficient value in the differential diagnosis of retroperitoneal masses. J Magn Reson Imaging 20: 735-742, 2004

15. Feugier P, Van Hoof A, Sebban C, Solal-Celigny P, Bouabdallah R, Fermé C, Christian B, Lepage E, Tilly H, Morschhauser F, et al: Long-term results of the R-CHOP study in the treatment of elderly patients with diffuse large B-cell lymphoma: A study by the Groupe d'Etude des Lymphomes de l'Adulte. J Clin Oncol 23: 4117-4126, 2005 